\title{
Tap Loading of Subcarrier Equalizers for Wireless Multicarrier Transceivers
}

\author{
Alexander M. Wyglinski, Member, IEEE, Martin Cudnoch, \\ Fabrice Labeau, Senior Member, IEEE, and Peter Kabal, Member, IEEE
}

\begin{abstract}
We present a novel algorithm for defining the lengths of subcarrier equalizers employed by wireless multicarrier transmission systems operating in frequency-selective fading channels. The equalizer lengths across the subcarriers are incrementally varied in a "greedy" fashion until the global cost function is below some prescribed threshold. By varying the equalizer lengths, the overall complexity of the equalization is constrained while the system meets a minimum error performance. Moreover, we investigate four strategies for terminating the proposed algorithm when an adequate number of equalizer taps have been allocated in this process. The results show that a system that employs variable-length equalizers defined by the proposed algorithm can achieve an improvement in error robustness of as much as an order of magnitude, relative to a system that employs constantlength equalizers with the same overall complexity.
\end{abstract}

Index Terms-Adaptive allocation, equalization, loading algorithms, multicarrier modulation.

\section{INTRODUCTION}

$\mathbf{T}$ O MEET the demand for reliable high-speed wireless access, multicarrier modulation is becoming the transmission technology of choice for several applications, e.g., wireless local area network systems [1], [2]. The primary advantage of multicarrier modulation is its ability to transmit information over frequency-selective fading channels using a "divide-andconquer" approach. Rather than transmitting data on a single carrier at a high data rate, information can be redistributed into several slower data streams, modulated on several different carriers, and simultaneously transmitted [3]. With respect to equalization and detection in multicarrier systems, the resulting receiver implementation complexity is lower since the frequency-selective fading channel has been transformed into a collection of approximately flat-fading subchannels [4].

Manuscript received November 3, 2005; revised August 29, 2006, March 5, 2007, and May 2, 2007. This work was supported in part by the Natural Sciences and Engineering Research Council of Canada and in part by Le Fonds Nature et Technologies du Quebec. This paper was presented in part at the 60th IEEE Vehicular Technology Conference, Los Angeles, CA, and in part at the 62nd IEEE Vehicular Technology Conference, Dallas, TX. The review of this paper was coordinated by Prof. J. Choi.

A. M. Wyglinski was with the Information and Telecommunication Technology Center, The University of Kansas, Lawrence, KS 66045-7576 USA. He is now with Department of Electrical and Computer Engineering, Worcester Polytechnic Institute, Worcester, MA 01609 USA (e-mail: alexw@ieee.org).

M. Cudnoch is with SR Telecom Inc., Montreal, QC H4S 1M5, Canada (e-mail: martin.cudnoch@gmail.com).

F. Labeau and P. Kabal are with the Department of Electrical and Computer Engineering, McGill University, Montreal, QC H3A 1Y1, Canada (e-mail: fabrice.labeau@mcgill.ca; peter.kabal@mcgill.ca).

Digital Object Identifier 10.1109/TVT.2007.905332
In a multicarrier receiver, when equalization is performed prior to the separation of the intercepted signal into its corresponding subcarriers by the analysis filter bank, the equalizer is referred to as a time-domain equalizer (TEQ). Conversely, an equalizer employed after the analysis filter bank is called a frequency-domain equalizer (FEQ). A FEQ would generally be applied per subcarrier to account for the magnitude and phase changes affecting that subcarrier [5]. As a result, a bank of FEQs would be employed by the receiver across all the subcarriers to compensate for any distortion.

There exists four major equalizer architectures for multicarrier systems: 1) a TEQ combined with a bank of single-tap FEQs [6]-[8]; 2) a bank of multitap FEQs [3], [6], [9]; 3) a TEQ filter bank [6]; and 4) a single linear system combining the TEQ, the analysis filter bank, and the bank of FEQs [6]. With TEQ implementations operating in the time domain of the multicarrier transceiver, there nevertheless exists several TEQ design methods that take into account the subchannel signalto-noise ratio (SNR) values when designing the finite-impulse response filter coefficients, i.e., these methods jointly optimize the time-frequency behavior of the equalized channel in designing the TEQ coefficients. Examples include the minimum InterSymbol Interference (ISI) method and the maximum bit rate method [10]. The minimum ISI is amenable to real-time fixed-point implementation in software and can be found in commercial asymmetric digital subscriber line modems.

Conversely, one of the advantages of performing equalization in the frequency domain is that the bank of FEQs can readily exploit the channel spectrum transformation achieved by multicarrier modulation. For a detailed quantitative comparison of the communication performance versus implementation complexity tradeoffs of several equalizer architectures, see the paper by Martin et al. [11]. One implementation of a frequencydomain equalizer architecture is the per-tone equalizer (PTEQ) approach [6], [12], which is designed to move the TEQ into the bank of single-tap FEQs to obtain a bank of multitap FEQs. This transformation is made possible by the fact that the TEQ, analysis filter bank, and FEQ are all linear systems in cascade. In this paper, we will focus only on equalizers belonging to the frequency-domain architecture category.

Although much work has gone into multitap FEQ design, there exists the potential for further improvements in the equalizer implementation. For example, in single-carrier systems, there exist several implementations where the tap lengths of the equalizer vary depending on some cost function or metric [13]-[16]. Extending this notion to multicarrier transceivers, the idea of nonuniformly varying the multitap FEQ length 
across the subcarriers, using a subcarrier equalizer tap loading algorithm, has also been proposed in several designs [17], [18]. Thus, the aim of tailoring equalizer lengths is efficient allocation of hardware resources based on when and where they are needed. With respect to adjusting the lengths of the subcarrier equalizers, deciding when an adequate number of taps have been allocated to each subcarrier is the task of the algorithm's termination strategy. Too many allocated taps will cause a reduction in distortion that does not justify the increase in computation time and complexity, whereas too few taps will give a poor performance. Therefore, the design of the termination strategy is important for an efficient implementation of the multitap FEQ.

In this paper, we present a novel algorithm for determining the lengths of the subcarrier frequency-domain equalizers for an indoor wireless multicarrier transceiver with perfect channel knowledge available at both the transmitter and the receiver. It improves upon our previous algorithm [17], [18] by choosing equalizer lengths that result in the overall distortion being below some prescribed threshold, rather than a distortion threshold on individual subcarriers. We also investigate four new termination strategies for the subcarrier equalizer tap loading algorithm that ensure that a sufficient number of equalizer taps have been allocated. Following a description of the multicarrier transceiver framework in Section II, we present the proposed subcarrier equalizer tap loading algorithm in Section III, along with details on the four termination strategies. Simulation results of multicarrier systems employing the proposed algorithm are then presented in Section IV, followed by some concluding remarks in Section V.

Notation: Vectors are denoted by lower case bold-faced letters. Matrices are defined by upper case bold-faced letters. Scalars are denoted by lower case letters. The superscript in parentheses (if present) indicates the subcarrier, whereas the subscript indicates either the sampling instants (vectors and scalars) or the matrix dimensions (matrices, except for upsampling and downsampling matrices). The superscripts $*$, $T$, and $H$ denote complex conjugation, transpose, and complex conjugation transpose, respectively.

\section{System SetuP}

A modified discrete Fourier transform (MDFT) filter bank transceiver [9] was employed in this paper since it provides a convenient framework for studying the proposed equalizer tap loading algorithm by using a more generic multicarrier transceiver design, i.e., the choice of prototype filter for the analysis/synthesis filter banks. For instance, the widely implemented orthogonal frequency-division multiplexing transceiver, which uses a discrete Fourier transform and its inverse for the analysis and synthesis filter banks, can be employed by the proposed tap loading algorithm (after the cyclic extension has been included in the transceiver design).

The general setup of this system is shown in Fig. 1, with a mathematical description provided in Appendix A. Note that an MDFT filter bank transceiver is designed to minimize the intercarrier interference resulting from phase offsets between the real and imaginary data components of the subcarriers

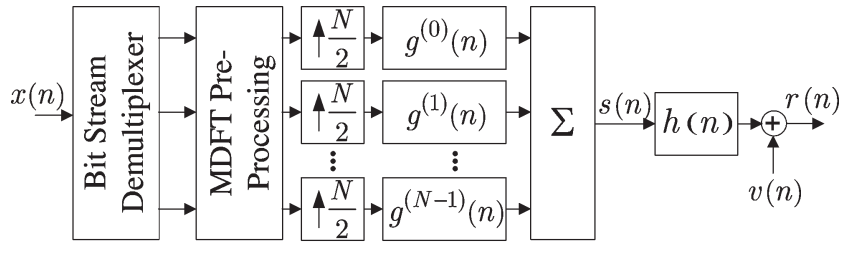

(a)

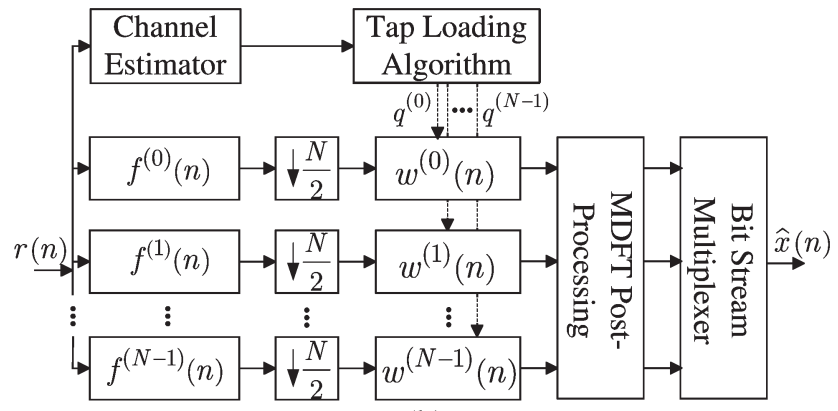

(b)

Fig. 1. Schematic of an MDFT multicarrier filter bank system performing subcarrier equalizer tap loading. (a) Transmitter with channel. (b) Receiver with PTEQs, channel estimator, and equalizer tap loading algorithm.

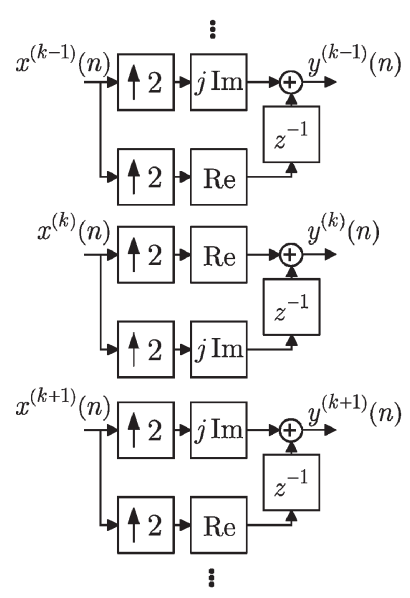

(a)
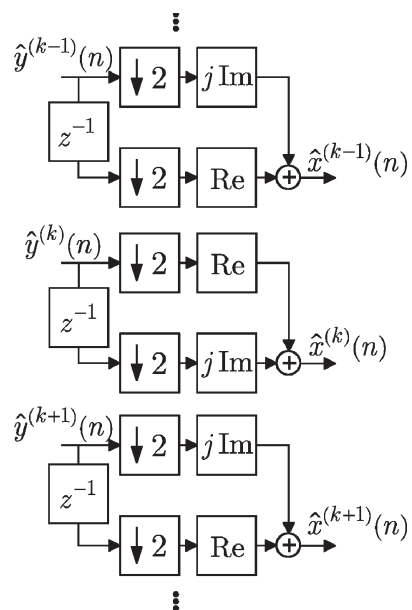

(b)
Fig. 2. Implementation of MDFT (a) preprocessing and (b) postprocessing components.

[9]. Moreover, to reduce sidelobe levels, a highly spectrally selective prototype filter can be employed for designing the synthesis and analysis filters.

The MDFT transceiver operates as follows: The high-speed complex input symbol stream $x(n)$ is demultiplexed into $N$ streams, with stream $i$ having $b_{i}$ bits per symbol epoch. The bit streams are modulated onto one of several signal constellations consisting of $M_{i}=2^{b_{i}}$ points. The outputs $x^{(i)}(n), i=$ $0, \ldots, N-1$, are then MDFT preprocessed, where $x^{(i)}(n)$ is upsampled by a factor of 2 , the real and imaginary components are separated, one of the components is delayed by one sample, and the components are recombined. The MDFT preprocessing is shown in Fig. 2(a). The outputs $y^{(i)}(n), i=0, \ldots, N-1$ are upsampled by a factor $N / 2$ and filtered by synthesis filters $g^{(i)}(n), i=0, \ldots, N-1$ before being summed together, yielding the composite transmit signal $s(n)$. 
This signal is transmitted across the channel, where the multipath propagation and additive noise are modeled with channel impulse response $h(n)$ and noise $v(n)$. The received signal $r(n)$ is separated into the $N$ subchannels using the analysis filters $f^{(i)}(n), i=0, \ldots, N-1$, and downsampled by a factor $N / 2$. Thus, the transfer function of the transceiver corresponding to the $k$ th subcarrier prior to equalization and MDFT postprocessing is given by

$$
\begin{array}{r}
\overline{\mathbf{y}}_{n, n-2 q^{(k)}+1}^{(k)}=\mathbf{T}_{d, R, 0} \mathbf{F}^{(k)} \mathbf{H} \sum_{l=0}^{N-1} \mathbf{G}^{(l)} \mathbf{T}_{u, R, D} \mathbf{y}_{n, n-2 L+1}^{(l)} \\
+\mathbf{T}_{d, R, 0} \mathbf{F}^{(k)} \mathbf{v}_{0,2 L R+D-P-S+2}
\end{array}
$$

where $\mathbf{y}_{n, n-2 L+1}^{(k)}, k=0, \ldots, N-1$ is the output vector of the MDFT preprocessing stage with length $2 L, \mathbf{T}_{u, R, D}$ is the upsampling matrix defined in (6), $\mathbf{G}^{(k)}$ is the convolution matrix of the synthesis filter $g^{(k)}(n)$ defined by $(7), \mathbf{F}^{(k)}$ is the convolution matrix of the analysis filter $f^{(k)}(n), \mathbf{H}$ is the convolution matrix of channel $h(n)$, and $\mathbf{v}_{0,2 L R+D-P-S+2}$ is the additive white Gaussian noise contribution of the channel. For details on the derivation of this transfer function, see Appendix A.

The channel estimator extracts channel information from $r(n)$, which is then used by the equalizer tap loading algorithm to determine the number of equalizer taps for subcarrier $i$, $q^{(i)}$, for $i=0, \ldots, N-1$. Channel estimation in multicarrier systems can be employed to determine the amount of magnitude and phase distortion introduced across the subcarriers of the transmitted signal. The estimation process can be achieved using either data-assisted [19], blind [20], or semiblind [20] approaches. As a result, this information could be used by the equalizer to reverse the effects of the channel on the received signal. Note that there exists several equalizer implementations that do not require the use of the channel estimate information [12], [21].

Given the tap lengths, the subcarrier signals are equalized using $w^{(i)}(n), i=0, \ldots, N-1$, MDFT postprocessed [see Fig. 2(b)], demodulated, and then multiplexed together to form the estimate $\hat{x}(n)$. In the next section, the process of determining the subcarrier equalizer lengths will be presented.

\section{SubCARrier Equalizer TAP LOAding Algorithm}

In a frequency-selective fading channel environment, the distortion introduced across the subcarriers is nonuniform. As a result, employing subcarrier equalizers of the same length is not an efficient use of hardware resources when attempting to reduce the overall distortion. Moreover, subcarriers with very poor performance and insufficient equalizer lengths will dominate the overall distortion of the system. Thus, Section III-A describes the proposed algorithm, which is designed to reduce the overall distortion of the system by assigning nonuniform equalizer lengths across the subcarriers. Knowing when "enough is enough" with respect to equalizer tap assignment is the topic of Section III-B.

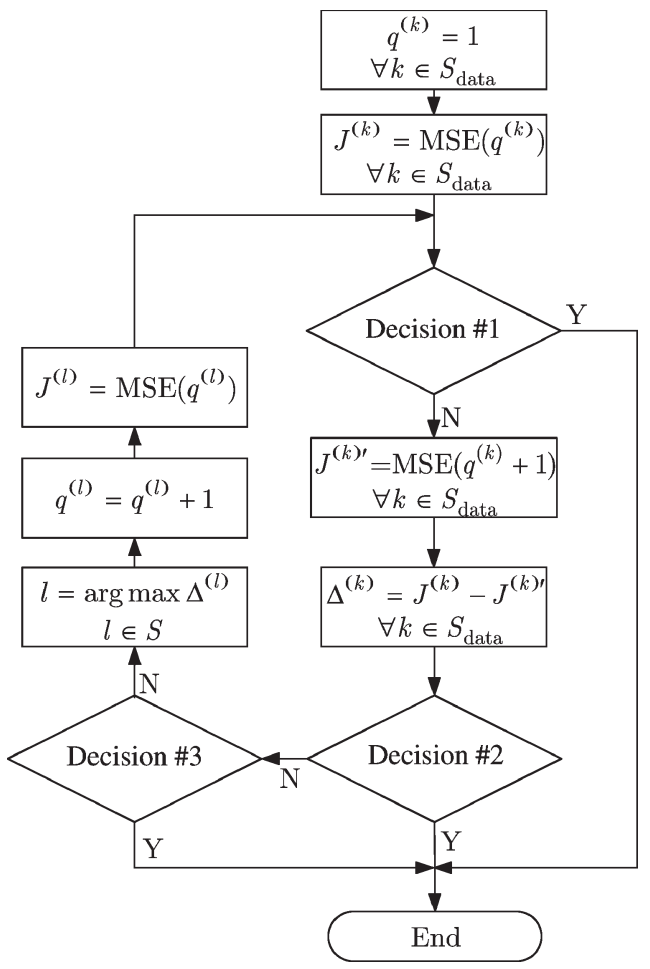

Fig. 3. Flow diagram of the proposed subcarrier equalizer tap loading algorithm.

\section{A. Proposed Algorithm}

The flow diagram of the proposed algorithm for obtaining the lengths of the subcarrier equalizers is shown in Fig. 3. Given a multicarrier system with $N$ subcarriers, there exists a subset of those subcarriers $S_{\text {data }}$ that are either data-bearing or pilot subcarriers, whereas the subcarriers not belonging to $S_{\text {data }}$ are "turned off" (i.e., nulled). The algorithm begins by setting the subcarrier equalizer lengths $q^{(k)}, k \in S_{\text {data }}$ to a length of one tap. Note that all equalizer weights in this paper are computed using a closed-form expression based on a minimum meansquare error (MMSE) cost function, i.e.

$$
\begin{aligned}
J^{(k)}= & \sigma_{y}^{2}- \\
& \operatorname{Re}\left\{\mathbf{p}_{y, r}^{(k) H} \mathbf{w}^{(k)}\right\}-\operatorname{Re}\left\{\mathbf{p}_{y, i}^{(k) H} \mathbf{w}^{(k)}\right\} \\
+ & \frac{1}{4}\left(\operatorname{Re}\left\{\mathbf{w}^{(k) H} \mathbf{R}_{y, r}^{(k)} \mathbf{w}^{(k)}\right\}+\operatorname{Re}\left\{\mathbf{w}^{(k) H} \mathbf{R}_{y, i}^{(k)} \mathbf{w}^{(k)}\right\}\right. \\
& \left.+\operatorname{Re}\left\{\mathbf{w}^{(k) H} \mathbf{R}_{y, r}^{(k) \prime} \mathbf{w}^{(k) *}\right\}-\operatorname{Re}\left\{\mathbf{w}^{(k) H} \mathbf{R}_{y, i}^{(k) \prime} \mathbf{w}^{(k) *}\right\}\right)
\end{aligned}
$$

where $\mathbf{w}^{(k)}$ represents the $k$ th subcarrier equalizer vector. Details regarding the mathematical expressions for the MMSE cost function and equalizer tap weights can be found in Appendices A and B. Using (2), the equalizer weights $w^{(k)}(n)$ that form equalizer vector $\mathbf{w}^{(k)}$ and the corresponding theoretical mean-square error (MSE) values $J^{(k)}$ for all the subcarriers belonging to $S_{\text {data }}$ are computed given the lengths $q^{(k)}$.

The proposed algorithm contains three decision blocks for terminating the allocation process once a sufficient number of 
equalizer taps have been assigned. Each block employs a different criterion in the decision process. Details regarding these criteria are presented in Section III-B. The first decision block that the proposed algorithm will encounter, i.e., Decision 1, judges whether a sufficient number of equalizer taps have been allocated. An example of such a decision would be a comparison between the mean of all $J^{(k)}$ values, i.e., $\bar{J}$, and a prescribed overall MSE threshold for the system, i.e., $J_{T}$. In this case, if $\bar{J} \leq J_{T}$, then none of the equalizer lengths needs to be increased to reduce $\bar{J}$, and the algorithm ends. However, if $\bar{J}>J_{T}$, then the algorithm needs to increase some of the lengths $q^{(k)}$ to satisfy $\bar{J} \leq J_{T}$.

Since the proposed algorithm operates in a "greedy" fashion [22], it incrementally increases the length of the subcarrier equalizer that maximizes the decrease in $\bar{J}$. Therefore, the algorithm computes the equalizer weights $w^{(k) \prime}(n)$ and corresponding theoretical MSE values $J^{(k) \prime}$ when the lengths are $q^{(k)}+1$ for all data-bearing subcarriers. The differences $\Delta^{(k)}=J^{(k)}-J^{(k) \prime}$ are computed, and the maximum difference is determined. The algorithm then encounters its second and third decision blocks, i.e., Decisions 2 and 3, for determining if a sufficient number of equalizer taps have been allocated to the system. The Decision 2 block can be used to check if there are any eligible subcarriers equalizer tap allocation. For instance, we can define set $S$ as $S \subset S_{\text {data }}$, which contains the subcarriers with $q^{(k)} \leq q_{\max }$, where $q_{\max }$ is a prescribed subcarrier equalizer length limit. If $S$ is empty, the algorithm breaks out and ends. Decision 3 block would check to see if it is worthwhile to continue allocating equalizer taps to the system. For example, the values of $\Delta^{(k)}, k \in S$, can be compared with a prescribed difference threshold $\Delta_{T}$. If $\max \left(\Delta^{(k)}\right) \leq \Delta_{T}$, then the algorithm breaks out and ends since the difference in the cost function due to an additional equalizer tap is small. On the other hand, if $\max \left(\Delta^{(k)}\right)>\Delta_{T}$, then the algorithm chooses subcarrier $l, l \in S$, which has the largest $\Delta^{(k)}$. The algorithm updates $q^{(l)}=q^{(l)}+1$ then computes $J^{(l)}$. Finally, $\bar{J} \leq J_{T}$ is compared, and the process repeats.

\section{B. Termination Strategies}

The proposed algorithm in Section III-A has three decision blocks to perform, i.e., Decisions 1-3 in Fig. 3, each of which are designed to end the algorithm once some prescribed criterion has been satisfied. Each of these steps forms a termination strategy of the algorithm and is primarily responsible for the adequate allocation of taps. These strategies can be designed to end the tap loading process for either a single subcarrier or the entire system. We will now cover four termination strategies that can be employed by the proposed algorithm.

1) Mean-Cost-Function-Based Strategy: This termination strategy, which can be employed in the Decision 1 block of Fig. 3, ends the loading algorithm once an adequate number of taps have been allocated such that the overall distortion is below some predefined limit. This is a global strategy since it requires information from all the subcarriers to make a decision and end the algorithm. Thus, the strategy ends the algorithm when

$$
\bar{J} \leq J_{T}
$$

since the mean cost function is above the minimum allowable level of quality for the transmission.

This strategy requires some advance knowledge of the channel and noise level to set $J_{T}$ to an appropriate value. If $J_{T}$ is below the noise floor, the loading algorithm will never satisfy (3), irrespective of the number of taps, i.e., the algorithm will never end. Conversely, if $J_{T}$ is set too high, the algorithm will end before an adequate number of taps have been allocated.

2) Mean-Cost-Function-Difference-Based Strategy: This termination strategy, which can be employed in the Decision 3 block of Fig. 3, operates by ending the entire loading process whenever the largest difference between the current and the subsequent subcarrier cost function is below some prescribed threshold. Therefore, this is a global strategy since it also requires information from all the subcarriers to make a decision and end the algorithm. The rationale behind this strategy is that the tap loading ends when the distortion decrease does not warrant an increase in implementation complexity. This strategy is equivalent to comparing the largest difference of the current and subsequent mean cost functions to some prescribed limit. The subsequent allocation is generated by adding an equalizer tap to the current allocation to the subcarrier, yielding the largest change in the cost function.

Mathematically, this strategy terminates the loading algorithm when

$$
\bar{\Delta}=\frac{\bar{J}-\bar{J}^{\prime}}{\bar{J}} \leq \Delta_{T}
$$

where $\bar{J}$ and $\bar{J}^{\prime}$ are the current and subsequent mean cost functions, and $\Delta_{T}$ is the prescribed mean cost function difference limit.

The advantage of using this strategy is that a percentage metric, rather than an absolute metric, is employed. As a result, prior knowledge of the channel conditions and system implementation is not required in choosing an appropriate value for $\Delta_{T}$. However, this strategy does not take into account the practical restrictions imposed by a hardware implementation, such as a limit on the amount of available memory or processing power. If threshold $\Delta_{T}$ is high, fewer taps will be allocated, resulting in the system's hardware resources being underused. On the other hand, a low value for $\Delta_{T}$ will result in longer equalizers, requiring more hardware to implement. These issues will be addressed in the next two sections.

3) Maximum-Taps-Per-Subcarrier-Based Strategy: Instead of ending the loading algorithm when some global criterion has been met, it may be more advantageous to stop allocating taps to specific subcarriers by limiting the individual subcarrier tap lengths. For instance, in Section III-A, we could limit the maximum number of taps per subcarrier to be below $q_{\max }$. If any of the subcarriers have reached $q_{\max }$, they would no longer be eligible to receive additional taps. As a result, this strategy only looks at the tap length of a specific subcarrier and does not require information from other subcarriers, although this strategy indirectly has a global effect since, in Decision 2 block of Fig. 3, the algorithm could be designed to end when all subcarriers have each reached the tap limit $q_{\max }$, resulting in $\mathcal{S}$ 
being empty since none of the subcarrier equalizers is available to accept any additional taps.

An advantage of this strategy is that the maximum lengths of equalizers are known in advance; thus, the hardware design can employ a fixed amount of resources for the equalizer implementation. Moreover, the maximum possible group delay of the equalizers will also be known. Finally, with an increase in the length of an equalizer, there is a linear increase in the number of computations. Thus, a hard limit on the equalizer length establishes an upper bound on both the total delay and the total number of operations required. The only disadvantage of this strategy is that it can only be used in combination with a second termination strategy. Otherwise, all equalizers would be of length $q_{\max }$.

4) Bank of Equalizer Taps Strategy: This termination strategy builds upon the strategy in Section III-B3 by imposing constraints on the maximum complexity of the hardware implementation. However, instead of limiting the number of taps per subcarrier, this strategy limits the total number of equalizer taps allocated. Thus, for the same hardware complexity, the loading algorithm has additional flexibility in allocating taps.

A finite pool of equalizer taps of size $q_{\text {tot }}$ is available to the loading algorithm when allocating across the data-bearing subcarriers of the system. Every time the length $q^{(k)}$ of a subcarrier equalizer is incremented, the pool of available taps is decremented. Once the pool is empty, no more taps are available, and the algorithm terminates. In other words, the algorithm continues to allocate until

$$
\sum_{k \in \mathcal{S}_{\text {data }}} q^{(k)}=q_{\text {tot }}
$$

in which case the algorithm ends. This termination strategy would be evaluated at the Decision 1 block of Fig. 3 . The only disadvantage of this strategy is that the worst case group delay of the subcarrier equalizers is larger, relative to the strategy in Section III-B3. Conversely, the greater degree of flexibility gained by the loading algorithm can result in allocations with a smaller overall distortion.

\section{Simulation Result}

We evaluated an MDFT filter bank multicarrier system [9] employing the proposed algorithm when operating in a frequency-selective fading environment modeled by the European Telecommunications Standards Institute (ETSI) HiperLAN/2 indoor channel responses [23]. The synthesis and analysis filters of the MDFT filter bank are modulated versions of a root-raised-cosine low-pass filter [24]. Many of the operating parameters of the system correspond to the IEEE Std. 802.11a [1], including the modulation schemes [binary phase-shift keying, quaternary phase-shift keying, square 16-state quadrature amplitude modulation (QAM), and square 64-state QAM (64-QAM)], subcarrier spacing, and operating frequency $(5 \mathrm{GHz})$. For straightforward comparison, the system did not employ channel coding. The input data to the transceiver were also not interleaved. MMSE equalizers were employed per subcarrier. Since we are evaluating the system in an indoor

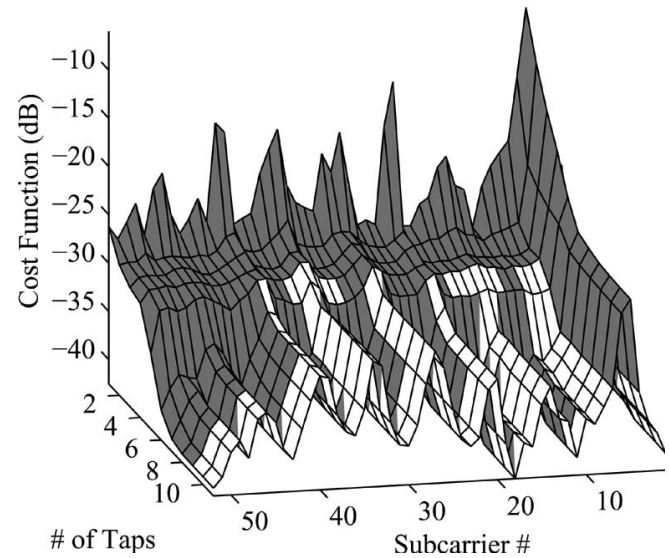

Fig. 4. Subcarrier MMSE as a function of subcarrier equalizer length using the ETSI HiperLAN/2 Channel B at $98 \mathrm{~dB}$.

environment, the channel is known not to rapidly vary over time. As a result, a set of equalizer weights and lengths computed by the proposed algorithm for a specific wireless channel can be employed for a sufficiently long period of time. Thus, the proposed algorithm can be realized in an actual hardware implementation, such as a software-defined radio, since it does not need to be frequently employed to update these weights and lengths. Moreover, we assume that the channel is perfectly known at the receiver, although, in practice, data-aided channel estimation techniques would be employed. Note that the errors resulting from the channel estimation process may potentially degrade the performance of the proposed tap loading algorithm, as indicated by similar equalizer design studies [25].

The subcarrier equalizer tap loading algorithm from Fig. 3 was employed, with the termination strategy of Section III-B4 used in the Decision 1 block. This strategy, rather than the strategy of Section III-B1, was employed to ensure that the allocation process meets the hardware requirements of the system. The termination strategies of Sections III-B2 and 3 were included in the Decisions 2 and 3 blocks. Unless stated otherwise, we chose $q_{\max }=20$ taps and $\Delta_{T}=10^{-4}$. The performance of the proposed algorithm when $q_{\text {tot }}=104$ taps and $q_{\text {tot }}=520$ taps was investigated with respect to distortion reduction, equalizer tap allocation, and bit error rate (BER) performance. Moreover, the impact on the BER performance of the system due to the choice of $\Delta_{T}$ is also studied, where $q_{\text {tot }}=520$ taps to ensure that the termination strategy of Section III-B4 is not responsible for ending the algorithm. Finally, all results were obtained via Monte Carlo simulations, where simulations ended after either the subcarrier with the fewest errors accumulated 100 errors or the overall accumulated error equaled $N \times 1000$.

\section{A. Cost Function versus the Number of Equalizer Taps}

The subcarrier MMSE distortion as a function of the number of equalizer taps is shown in Fig. 4 for all 52 data-bearing subcarriers, given the ETSI HiperLAN/2 Channel B at an SNR of $98 \mathrm{~dB}$. Note that the dark regions of the surface plot indicate the equalizer tap allocation given the channel and SNR value. The results show that the MMSE monotonically decreases as 


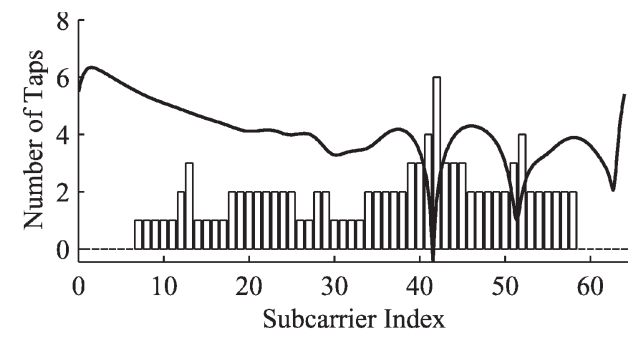

(a)

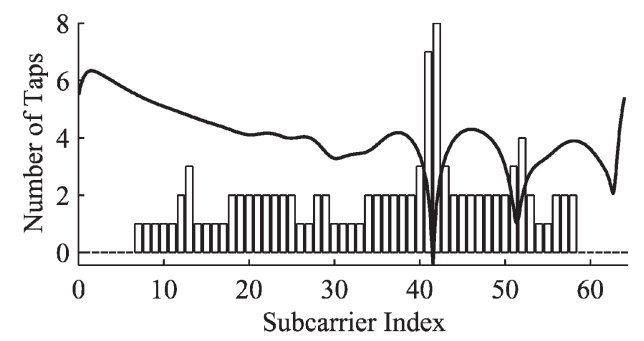

(b)

Fig. 5. Equalizer tap allocation given the ETSI HiperLAN/2 Channel A (superimposed) for different SNR values and $q_{\text {tot }}=104$ taps. (a) Equalizer tap allocation at an SNR of $31.7 \mathrm{~dB}$. (b) Equalizer tap allocation at an SNR of $38.7 \mathrm{~dB}$.

the number of equalizer taps increases. However, the MMSE versus the number of equalizer taps is not a strictly convex function for all subcarriers since the equalizer taps are constrained to uniform sampling locations, which might not correspond to an optimal solution. Therefore, each additional tap to a subcarrier equalizer may result in a decrease in distortion. However, that decrease may not be smaller than the previous allocations for that subcarrier.

During our simulations, it was noticed that, for the small bank of $q_{\text {tot }}=104$ taps, the limiting factor of the loading algorithm was the size of the bank of equalizer taps. This translates into an early stop in the allocation. Conversely, when $q_{\mathrm{tot}}=520$ taps is used, the $\Delta_{T}$ limit will be the prevalent mode of termination for the loading algorithm. As a result, the algorithm ends when a substantial number of taps have been allocated. Finally, the subcarrier tap limit of $q_{\max }=20$ taps was seldom employed (e.g., three subcarriers out of all the databearing subcarriers for all five ETSI channels ever reached that limit).

\section{B. Subcarrier Tap Allocation}

Several observations can be made regarding the equalizer tap allocations for two different SNR values, such as allocations at 31.7 and $38.7 \mathrm{~dB}$, given the ETSI HiperLAN/2 Channel A, as shown in Fig. 5. First, when a spectral null is present, the loading algorithm will try to add more equalizer taps to invert the effects of the distortion in that subcarrier. For instance, the algorithm has allocated the largest number of taps per subcarrier to subcarrier 42 and its adjacent subcarriers, which are in the vicinity of a spectral null. However, if the spectral null is too steep and the incremental gain of additional taps on the cost function is small, the algorithm will stop allocating taps to that subcarrier. Nevertheless, it is advantageous to employ

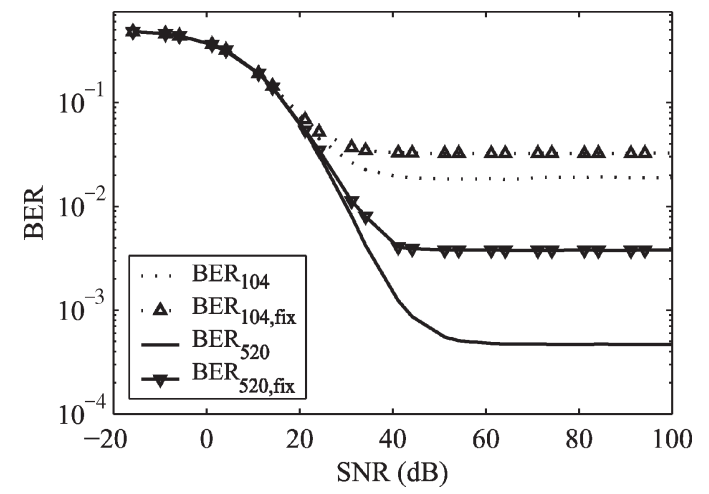

Fig. 6. BER curves for fixed-tap-length (with triangular markers) and tapallocated (without triangular markers) multicarrier systems employing square 64-QAM modulation across all subcarriers given the ETSI Channel E. Results are shown for $q_{\mathrm{tot}}=104$ taps (dotted lines) and $q_{\mathrm{tot}}=520$ taps (solid lines).

variable-length subcarrier equalizers, with each subcarrier allocated enough equalizer taps to achieve an overall increase in error robustness, relative to a system employing uniform-length subcarrier equalizers with the same overall implementation complexity.

When $q_{\mathrm{tot}}=520$ taps, the number of taps allocated increases, relative to an increase in the SNR. Moreover, in several subcarriers, the tap lengths may also increase. This is due to the lowering of the noise floor, leaving the frequency-selective fading channel as the predominant form of distortion. When $q_{\mathrm{tot}}=$ 104 taps, it is observed that the tap allocations are rearranged at different SNR values. For instance, several subcarriers around subcarrier 42 may gain taps, whereas other subcarriers lose taps after an increase in SNR. This rearrangement occurs when the distortion-versus-tap-number function is not convex, due to the suboptimal placement of taps in an equalizer.

\section{BER Results}

The BER results for multicarrier systems using a set of subcarrier equalizers of variable length (determined by the proposed algorithm) and a set of constant-length subcarrier equalizers (determined by taking the total number of taps allocated to the entire transceiver by the proposed algorithm and dividing this value by the total number of data-bearing subcarriers) are presented in Fig. 6. The systems employed 64-QAM signal constellations per subcarrier and operated in the ETSI HiperLAN/2 Channel E. At low SNR values, tap allocation is ineffective when the noise floor is high. This means that the distortion-versus-tap-number function will flatten out early in the curve and that additional taps will not improve performance. As a result, not many taps are allocated, and the performance is comparable to the fixed-length scheme. It should be pointed out that, even though IEEE Std. 802.11a systems possess a minimum SNR requirement of $6 \mathrm{~dB}$, they can have SNR values in the range of $25-60 \mathrm{~dB}$, which is relatively high.

As the SNR increases, more taps are allocated as the noise floor lowers. Consequently, the channel becomes the principal source of distortion, which can be more effectively handled by the equalizers. Therefore, more equalizer taps are employed since their addition significantly improves the overall 


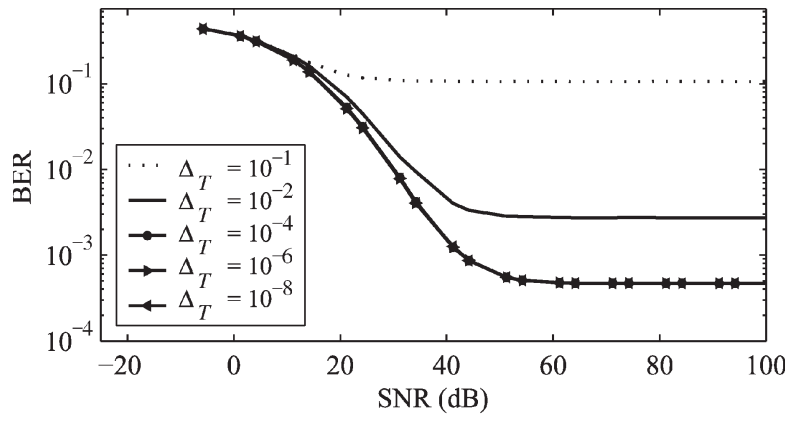

(a)

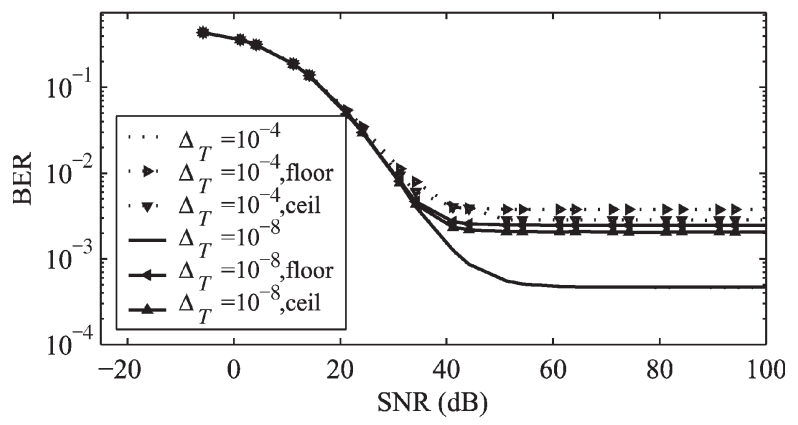

(b)

Fig. 7. BER performance, relative to $\Delta_{\mathrm{TH}}$ ETSI HiperLAN/2 Channel E. (a) BER performance for the variable-length equalizer for different values of $\Delta_{\mathrm{TH}}$. (b) BER performance comparison between variable-length equalizers and their corresponding fixed-length schemes. The "floor" and "ceil" suffixes correspond to fixed equalizers with $q_{\mathrm{FLOOR}}$ and $q_{\mathrm{CEIL}}$ taps, respectively.

performance of the system. Moreover, with more equalizer taps available to the proposed algorithm, the system employing $q_{\text {tot }}=520$ taps has drastically better BER performance, relative to a system with $q_{\text {tot }}=104$ taps at high SNR values starting at $20 \mathrm{~dB}$. In fact, given $q_{\mathrm{tot}}=520$ taps, the improvement in BER for a system employing the proposed algorithm is as much as an order of magnitude, relative to a system with fixed-length equalizers and comparable overall complexity. Finally, relative to systems using uniform-tap-length subcarrier equalizers with an equivalent total number of taps, employing a rounded average number of taps per equalizer, the system employing tap loading algorithms performs better.

\section{Impact of the Cost Function Difference Threshold $\Delta_{T}$ on BER Performance}

We studied the BER performance of the system for values of $\Delta_{T}$ ranging from $10^{-1}$ down to $10^{-8}$. As expected, with decreasing values of $\Delta_{T}$, the number of taps monotonically increases. However, this increase in complexity does not necessarily translate to a better performance. Fig. 7(a) displays the BER curves versus the SNR for different values $\Delta_{T}$. When $\Delta_{T}$ is large, lowering the threshold will also significantly lower the BER floor. However, the decrease in BER reaches a plateau for $\Delta_{T}=10^{-4}$. Setting an even lower threshold will only generate trivial improvements in BER performance.

Fig. 7(b) shows the comparison in terms of BER between the fixed- and variable-length equalizers for the ETSI HiperLAN/2
Channel E. Note that the curves for variable-length schemes with $\Delta_{T}=10^{-4}$ and $\Delta_{T}=10^{-8}$ overlap. As the complexity of the equalizers grows beyond $\Delta_{T}=10^{-4}$, the difference in probability of error between the variable- and fixed-length schemes becomes smaller. Thus, for very small values of $\Delta_{T}$, the two will yield the same performance. At this stage, fixedlength equalizers are preferable, because they are simpler to compute.

However, the plateau reached at low $\Delta_{T}$ might be a local minimum, instead of a global one. In this case, lowering the threshold even more will allow the algorithm to "bypass" the local minimum and converge to the optimum solution. A $\Delta_{T}$ that is low enough to achieve this might however make the complexity of the system computationally prohibitive. In the case of this experiment, even setting $\Delta_{T}=10^{-8}$ did not improve the performance of the channels.

Thus, from a system designer point of view, setting $\Delta_{T}=$ $10^{-4}$ would be the sensible choice for every Hiperlan/2 ETSI channel tested.

\section{CONCLUSION}

A novel loading algorithm for defining the lengths of subcarrier frequency-domain equalizers is proposed. Using the proposed algorithm to tailor the lengths of the subcarrier equalizers to the channel conditions, the error robustness of the system is substantially enhanced, relative to systems employing samelength subcarrier equalizers with equivalent overall complexity. Moreover, this enhancement comes at the cost of a small increase in implementation complexity due to the incorporation of the algorithm in the system. Note that the incremental nature and objective of the proposed algorithm help ensure that every equalizer tap allocated makes the largest possible decrease in overall distortion per iteration.

The importance of algorithm termination was also investigated. In particular, we focused on four termination strategies that can be employed by the proposed algorithm to ensure that a sufficient number of equalizer taps are allocated. Furthermore, we studied the relationship between the total number of taps available for allocation and system error robustness. It was shown that, with more taps available, the error robustness can significantly increased.

\section{APPENDIX A System TRANSFER FUnCTION}

The system transfer function of our MDFT filter bank multicarrier system is derived using a similar framework as in our previous work [17], [18]. Referring to Figs. 1 and 2 as well as Section II, the modulated data streams $x^{(i)}(n), i=$ $0, \ldots, N-1$ are defined for each subcarrier as a vector

$$
\mathbf{x}_{n, n-L+1}^{(k)}=\left[x^{(k)}(n) \quad \ldots \quad x^{(k)}(n-L+1)\right]^{T}
$$

for $k=0, \ldots, N-1$ and of length $L$. These vectors are then used as inputs to the MDFT preprocessing stage of the system, the outputs of which are the vectors $\mathbf{y}_{n, n-2 L+1}^{(k)}$, $k=0, \ldots, N-1$, with length $2 L$. These outputs are then 
upsampled using a $(2 L R+D) \times(2 L)$ upsampling matrix $\mathbf{T}_{u, R, D}$, which is defined as

$$
\left.\mathbf{T}_{u, R, D}=\left[\begin{array}{cccc}
0 & 0 & \cdots & 0 \\
\vdots & \vdots & \ddots & \vdots \\
0 & 0 & \cdots & 0 \\
1 & 0 & \cdots & 0 \\
0 & 0 & \cdots & 0 \\
\vdots & \vdots & \ddots & \vdots \\
0 & 0 & \cdots & 1
\end{array}\right]\right\}_{R+D-1}
$$

where $R$ is the sampling rate, and $D$ is the delay. Each column of $\mathbf{T}_{u, R, D}$ possesses a single nonzero element, with no more than one nonzero element per row. The rows containing nonzero elements are separated by $R-1$ rows that only possess zerovalued elements. As a result, when $\mathbf{T}_{u, R, D}$ is multiplied with the vector $\mathbf{y}_{n, n-2 L+1}^{(k)}, R-1$ zeros are inserted between each element of $\mathbf{y}_{n, n-2 L+1}^{(k)}$, thus upsampling it. In this case, the sampling rate is $R=N / 2$ since, combined with the MDFT preprocessing stage, which performs an upsampling by a factor of 2, the overall upsampling rate is $N$ (i.e., critically sampled). Matrix $\mathbf{T}_{u, R, D}$ also accounts for the total delay introduced at different stages of the transceiver and channel by including $D$ rows possessing only zero-valued elements at the top of the matrix. The delay is $D=2 \operatorname{round}(\tau)+\operatorname{round}\left(\tau_{\mathrm{ch}}\right)$, where $\tau$ is the group delay of the synthesis or analysis filter, $\tau_{\mathrm{ch}}$ is the group delay of the channel, and round (.) rounds the input value to the nearest integer. By sufficiently zero-padding the transmitted vector, the mathematical analysis can adequately compensate for the effect of the total group delay introduced by the system [26].

The upsampled signals are then filtered by the synthesis filters $\mathbf{g}_{0, P-1}^{(k)}, k=0, \ldots, N-1$ of length $P$. The filtered signals are then summed together and transmitted across the channel, with an impulse response $\mathbf{h}_{0, S-1}$ of length $S$. The received signal is decomposed into $N$ subcarriers using the analysis filters $\mathbf{f}_{0, P-1}^{(k)}, k=0, \ldots, N-1$ of length $P$ before being downsampled by the $\left(2 q^{(k)}\right) \times(2 L R+D-2 P-S+3)$ downsampling matrix $\mathbf{T}_{d, N / 2,0}=\mathbf{T}_{u, N / 2,0}^{T}$, where $2 q^{(k)}$ is the length of the fractionally spaced MMSE equalizer $\mathbf{w}_{0,2 q^{(k)}-1}^{(k)}$, $k=0, \ldots, N-1$.

Filtering is performed in this derivation using convolution matrices [17], [18]. Therefore, we can represent $\mathbf{g}_{0, P-1}^{(k)}$ as an $(2 L R+D-P+1) \times(2 L R+D)$ convolution matrix, i.e.,

$$
\mathbf{G}^{(k)}=\left[\begin{array}{cccc}
\mathbf{g}_{0, P-1}^{(k) T} & 0 & \cdots & 0 \\
0 & \mathbf{g}_{0, P-1}^{(k) T} & \cdots & 0 \\
\vdots & \vdots & \ddots & \vdots \\
0 & 0 & \cdots & \mathbf{g}_{0, P-1}^{(k) T}
\end{array}\right]
$$

Furthermore, the channel $\mathbf{h}_{0, S-1}$ and the $k$ th analysis filter $\mathbf{f}_{0, P-1}^{(k)}$ can be represented as $(2 L R+D-P-S+$ $2) \times(2 L R+D-P+1)$ and $(2 L R+D-2 P-S+3) \times$
$(2 L R+D-P-S+2)$ convolution matrices $\mathbf{H}$ and $\mathbf{F}^{(k)}$, respectively.

The transfer function corresponding to the $k$ th subcarrier prior to the MDFT postprocessing is given by (1). To compensate for the distortion added to $\overline{\mathbf{y}}_{n, n-2 q^{(k)}+1}^{(k)}$ by the channel, as well as the synthesis and analysis filters, $2 q^{(k)}$-tap fractionally spaced MMSE equalizers $\mathbf{w}_{0,2 q^{(k)}-1}^{(k)}, k=0, \ldots, N-1$, are employed before performing MDFT postprocessing. Note that the taps for each equalizer are located at uniform sampling instants. Thus, the equalized vector becomes

$$
\hat{y}^{(k)}(n)=\mathbf{w}_{0,2 q^{(k)}-1}^{(k) H} \overline{\mathbf{y}}_{n, n-2 q^{(k)}+1}^{(k)}
$$

where the output is a scalar value at sampling instant $n$. Although other closed-form derivations for MDFT equalization do exist in the literature [9], we present our derivation in the next section for the MMSE cost function at the output of the MDFT postprocessing and equalization stages since the formulation conducted within this paper is differently done, relative to these other derivations, e.g., convolution matrices and sampling matrices are extensively employed in this paper.

\section{APPENDIX B}

MMSE EQUALIZER DERIVATION

Since the desired real and imaginary information of $\overline{\mathbf{y}}_{n, n-2 q^{(k)}+1}^{(k)}$ are $90^{\circ}$ out of phase with each other and alternatively occur, we must consider reducing the distortion of the real and imaginary components separately at specific sampling instants.

Therefore, the MSE cost function of the desired real and imaginary components can be defined as

$$
\begin{aligned}
& J^{(k)}= \frac{1}{2} E\left\{\left|\operatorname{Re}\left(y^{(k)}(2 m)-\hat{y}^{(k)}(2 m)\right)\right|^{2}\right\} \\
&+\frac{1}{2} E\left\{\left|\operatorname{Im}\left(y^{(k)}(2 m+1)-\hat{y}^{(k)}(2 m+1)\right)\right|^{2}\right\} \\
&= \frac{1}{8} E\left\{\mid y^{(k)}(2 m)-\hat{y}^{(k)}(2 m)\right. \\
&\left.+y^{(k) *}(2 m)-\left.\hat{y}^{(k) *}(2 m)\right|^{2}\right\} \\
&+ \frac{1}{8} E\left\{\mid y^{(k)}(2 m+1)-\hat{y}^{(k)}(2 m+1)\right. \\
&\left.\quad-y^{(k) *}(2 m+1)+\left.\hat{y}^{(k) *}(2 m+1)\right|^{2}\right\}
\end{aligned}
$$

where, without loss in generality, $n=2 m$ corresponds to the desired sampling instants for the real information in subcarrier $k$, whereas $n=2 m+1$ defines the desired sampling instants for the imaginary data. 
Expanding (9) and employing (8) yield (2) after some algebraic manipulation, where $\mathbf{w}^{(k)}=\mathbf{w}_{0,2 q^{(k)}-1}^{(k)}$ and $\sigma_{y}^{2}=$ $E\left\{y^{(k)}(2 m) y^{(k) *}(2 m)\right\}=E\left\{y^{(k)}(2 m+1) y^{(k) *}(2 m+1)\right\}$.

Moreover, we have

$$
\begin{array}{r}
\mathbf{p}_{y, t}^{(k) H} \mathbf{w}^{(k)}=E\left\{y^{(k)}(l) \hat{y}^{(k) *}(l)\right\} \\
\mathbf{w}^{(k) H} \mathbf{R}_{y, t}^{(k)} \mathbf{w}^{(k)}=E\left\{\hat{y}^{(k)}(l) \hat{y}^{(k) *}(l)\right\} \\
\mathbf{w}^{(k) H} \mathbf{R}_{y, t}^{(k) \prime} \mathbf{w}^{(k) *}=E\left\{\hat{y}^{(k)}(l) \hat{y}^{(k)}(l)\right\}
\end{array}
$$

where $l=2 m$ for $t=r$, and $l=2 m+1$ for $t=i$.

To obtain the fractionally spaced MMSE equalizer weights that minimize the cost function for subcarrier $k, J_{\min }^{(k)}$, we employ the Wirtinger derivative [27] given by

$$
\frac{\partial}{\partial \mathbf{w}^{(k) *}}=\frac{1}{2}\left[\begin{array}{c}
\frac{\partial}{\partial w_{R}^{(k)}(0)}+j \frac{\partial}{\partial w_{I}^{(k)}(0)} \\
\frac{\partial}{\partial w_{R}^{(k)}(1)}+j \frac{\partial}{\partial w_{I}^{(k)}(1)} \\
\vdots \\
\frac{\partial}{\partial w_{R}^{(k)}\left(2 q^{(k)}-1\right)}+j \frac{\partial}{\partial w_{I}^{(k)}\left(2 q^{(k)}-1\right)}
\end{array}\right]
$$

where $w_{R}^{(k)}(m)$ and $w_{I}^{(k)}(m)$ are the real and imaginary components of $w^{(k)}(m)$ to (2). This yields

$$
\begin{aligned}
\frac{\partial J^{(k)}}{\partial \mathbf{w}^{(k) *}}= & -4\left(\mathbf{p}_{y, r}^{(k)}+\mathbf{p}_{y, i}^{(k)}\right)+2\left(\mathbf{R}_{y, r}^{(k)}+\mathbf{R}_{y, i}^{(k)}\right) \mathbf{w}^{(k)} \\
& +2\left(\mathbf{R}_{y, r}^{(k) \prime}-\mathbf{R}_{y, i}^{(k) \prime}\right) \mathbf{w}^{(k) *} \\
= & 0
\end{aligned}
$$

which can be equivalently written as

$$
2\left(\mathbf{p}_{y, r}^{(k)}+\mathbf{p}_{y, i}^{(k)}\right)=\left(\mathbf{R}_{y, r}^{(k)}+\mathbf{R}_{y, i}^{(k)}\right) \mathbf{w}^{(k)}+\left(\mathbf{R}_{y, r}^{(k) \prime}-\mathbf{R}_{y, i}^{(k) \prime}\right) \mathbf{w}^{(k) *} .
$$

To solve for the equalizer weights, we arrange (11) into a block matrix expression $\mathbf{A w}=2 \mathbf{p}$, i.e.,

$$
\left[\begin{array}{ll}
\mathbf{A}_{11} & \mathbf{A}_{12} \\
\mathbf{A}_{21} & \mathbf{A}_{22}
\end{array}\right]\left[\begin{array}{l}
\operatorname{Re}\left(\mathbf{w}^{(k)}\right) \\
\operatorname{Im}\left(\mathbf{w}^{(k)}\right)
\end{array}\right]=2\left[\begin{array}{c}
\operatorname{Re}\left(\mathbf{p}_{y, r}^{(k)}+\mathbf{p}_{y, i}^{(k)}\right) \\
\operatorname{Im}\left(\mathbf{p}_{y, r}^{(k)}+\mathbf{p}_{y, i}^{(k)}\right)
\end{array}\right]
$$

where

$$
\begin{aligned}
& \mathbf{A}_{11}=\operatorname{Re}\left(\mathbf{R}_{y, r}^{(k)}+\mathbf{R}_{y, i}^{(k)}\right)+\operatorname{Re}\left(\mathbf{R}_{y, r}^{(k) \prime}-\mathbf{R}_{y, i}^{(k) \prime}\right) \\
& \mathbf{A}_{12}=-\operatorname{Im}\left(\mathbf{R}_{y, r}^{(k)}+\mathbf{R}_{y, i}^{(k)}\right)+\operatorname{Im}\left(\mathbf{R}_{y, r}^{(k) \prime}-\mathbf{R}_{y, i}^{(k) \prime}\right) \\
& \mathbf{A}_{21}=\operatorname{Im}\left(\mathbf{R}_{y, r}^{(k)}+\mathbf{R}_{y, i}^{(k)}\right)+\operatorname{Im}\left(\mathbf{R}_{y, r}^{(k) \prime}-\mathbf{R}_{y, i}^{(k) \prime}\right) \\
& \mathbf{A}_{22}=\operatorname{Re}\left(\mathbf{R}_{y, r}^{(k)}+\mathbf{R}_{y, i}^{(k)}\right)-\operatorname{Re}\left(\mathbf{R}_{y, r}^{(k) \prime}-\mathbf{R}_{y, i}^{(k) \prime}\right) .
\end{aligned}
$$

The equalizer weights are then determined by solving $\mathbf{w}=$ $2 \mathbf{A}^{-1} \mathbf{p}$, where the inverse of the block matrix $\mathbf{A}$ is [28]

$$
\mathbf{A}^{-1}=\left[\begin{array}{cc}
\mathbf{A}_{11}^{-1}+\mathbf{A}_{11}^{-1} \mathbf{A}_{12} \mathbf{S}^{-1} \mathbf{A}_{21} \mathbf{A}_{11}^{-1} & -\mathbf{A}_{11}^{-1} \mathbf{A}_{12} \mathbf{S}^{-1} \\
-\mathbf{S}^{-1} \mathbf{A}_{21} \mathbf{A}_{11}^{-1} & \mathbf{S}^{-1}
\end{array}\right]
$$

and $\mathbf{S}=\mathbf{A}_{22}-\mathbf{A}_{21} \mathbf{A}_{11}^{-1} \mathbf{A}_{12}$ is its Schur complement.

Note that the value of $q^{(k)}$ is determined by the proposed algorithm of Section III-A.

\section{APPENDIX C \\ COMPLEXITY ANALYSIS}

We observe from the flow diagram of Fig. 3 that most of the computational complexity contained within the proposed tap loading algorithm is located at the steps where the MSE is computed per subcarrier. In this paper, the value of $J^{(k)}$, $k=0, \ldots, N-1$, is determined by initially evaluating the overall system transfer function of (1). As a result, the number of complex multiplications and additions required to determine $\overline{\mathbf{y}}_{n, n-2 q^{(k)}+1}^{(k)}$ is given by

$$
\begin{aligned}
& N_{\text {mults }}\left(\overline{\mathbf{y}}_{n, n-2 q^{(k)}+1}^{(k)}\right) \\
& =N \cdot\left(2 L^{2}+4 L^{2} R+2 L D\right. \\
& \left.\quad \quad+(2 L R+D)^{2}-(2 L R+D) \cdot(P-1)\right) \\
& \quad+(2 L R+D-P+1)^{2}-(2 L R+D-P+1) \cdot(S-1) \\
& +2 \cdot\left((2 L R+D-P-S+2)^{2}\right. \\
& \quad \quad-(2 L R+D-P-S+2) \cdot(P-1)) \\
& \quad+2 \cdot\left((2 L R+D-2 P-S+3) \cdot 2 q^{(k)}\right) \\
& N_{\text {adds }}\left(\overline{\mathbf{y}}_{n, n-2 q^{(k)}+1}^{(k)}\right) \\
& =N \cdot\left(2 L^{2}-2 L+4 L^{2} R+2 L D-2 L R-D\right) \\
& +N \cdot\left((2 L R+D-1)^{2}-(2 L R+D-1) \cdot(P-2)\right) \\
& +(2 L R+D-P)^{2}-(2 L R+D-P) \cdot(S-2) \\
& +2 \cdot\left((2 L R+D-P-S+1)^{2}\right. \\
& \quad \quad-(2 L R+D-P-S+1) \cdot(P-2)) \\
& \quad+\left((2 L R+D-2 P-S+2) \cdot 2 q^{(k)}\right)
\end{aligned}
$$

where $N$ is the total number of subcarriers, $L$ is the length of the input sequence to the MDFT filter bank multicarrier system, $R=N / 2$ is the upsampling factor after the input sequence is upsampled by a factor of $2, P$ is the length of the analysis and synthesis filters, $S$ is the length of the channel, $D$ is the amount of delay incurred by the system transfer function, and $q^{(k)}$ is the length of the equalizer on subcarrier $k$. We observe in the preceding expressions that the dominant term is $\mathcal{O}\left((L R+D)^{2}\right)$. 
Once the system transfer function has been obtained, the other major source of computational complexity when determining the MSE value for a subcarrier equalizer $k$ occurs when solving for the matrix $\mathbf{A}^{-1}$. Given that the matrices used in (13), such as $\mathbf{A}_{11}, \mathbf{A}_{12}, \mathbf{A}_{21}, \mathbf{A}_{22}$, or $\mathbf{S}$, possess dimensions of $\left(2 q^{(k)}\right) \times\left(2 q^{(k)}\right)$, the multiplication of two such matrices will result in $n_{\text {mults }}=\left(2 q^{(k)}\right)^{3}$ real multiplications and $n_{\text {adds }}=$ $\left(2 q^{(k)}\right)^{2} \cdot\left(2 q^{(k)}-1\right)$ real additions. Thus, the number of real multiplications and additions required to compute the four elements of $\mathbf{A}^{-1}$ is given by

$$
\begin{aligned}
N_{\text {mults }}\left(\mathbf{A}^{-1}\right) & =\left[\begin{array}{cc}
4 n_{\text {mults }} & 2 n_{\text {mults }} \\
2 n_{\text {mults }} & --
\end{array}\right] \\
N_{\text {adds }}\left(\mathbf{A}^{-1}\right) & =\left[\begin{array}{cc}
4 n_{\text {adds }}+\left(2 q^{(k)}\right)^{2} & 2 n_{\text {adds }} \\
2 n_{\text {adds }} & --
\end{array}\right]
\end{aligned}
$$

where the computation of matrix $\mathbf{S}$ needs $2 n_{\text {mults }}^{2}$ multiplications and $2 n_{\text {adds }}+\left(2 q^{(k)}\right)^{2}$ additions. Note that the inverse of any $\left(2 q^{(k)}\right) \times\left(2 q^{(k)}\right)$ matrix in (13) has an order of complexity of $\mathcal{O}\left(\left(2 q^{(k)}\right)^{3}\right)$.

In Appendices A and B, the optimum formula for the equalizer weights have been derived. As a result, this paper can demonstrate the concept of the proposed tap loading algorithm. Overall, this method remains rather complex, particularly because these derivations rely on a series of matrix inverses, which, in practice, should be avoided due to numerical stability issues (which depends on the condition number), computational complexity, and the mismatch to a cost-effective fixed-point implementation. Again, the aim of the this paper is to demonstrate the concept of tap loading in multicarrier systems, and complexity reduction issues will be the subject of forthcoming research.

In future work, we will carry the derivation further to develop an adaptive framework that is free of matrix inverses and decompositions. Thus, the computational complexity of the proposed tap loading algorithm will be reduced and eventually approach that of other adaptive length equalization techniques found in the literature [15], [16].

\section{ACKNOWLEDGMENT}

The authors would like to thank the anonymous reviewers for their careful reading of this paper as well as their suggestions and comments.

\section{REFERENCES}

[1] Wireless LAN Medium Access Control (MAC) and Physical Layer (PHY) Specifications: High-Speed Physical Layer in the $5 \mathrm{GHz}$ Band, IEEE Std. 802.11a, Nov. 1999.

[2] Broadband Radio Access Networks (BRAN): HIPERLAN Type 2; Physical (PHY) Layer, ETSI TS 101 475, Dec. 2001

[3] J. Louveaux, "Filter bank based multicarrier modulation for xDSL transmission," Ph.D. dissertation, Univ. Catholique de Louvain, Louvain-la-Neuve, Belgium, May 2000.

[4] J. A. C. Bingham, "Multicarrier modulation for data transmission: An idea whose time has come," IEEE Commun. Mag., vol. 28, no. 5, pp. 5-14, Apr. 1990
[5] T. Pollet, M. Peeters, M. Moonen, and L. Vandendorpe, "Equalization for DMT based broadband modems," IEEE Commun. Mag., vol. 38, no. 5, pp. 106-113, May 2000.

[6] R. K. Martin, K. Vanbleu, M. Ding, G. Ysebaert, M. Milosevic, B. L. Evans, M. Moonen, and C. R. Johnson, Jr., "Unification and evaluation of equalization structures and design algorithms for discrete multitone modulation systems," IEEE Trans. Signal Process., vol. 53, no. 10, pp. 3880-3894, Oct. 2005.

[7] K. Vanbleu, G. Ysebaert, G. Cuypers, and G. Leus, "Adaptive bitrate maximizing TEQ designs for DMT-based systems," in Proc. IEEE Int. Conf. Acoust., Speech, Signal Process., Montreal, QC, Canada, May 2004 vol. 4, pp. 1057-1060.

[8] N. Al-Dhahir and J. M. Cioffi, "Optimum finite-length equalization for multicarrier transceivers," IEEE Trans. Commun., vol. 44, no. 1, pp. 5664, Jan. 1996.

[9] T. Wiegand and N. J. Fliege, "Equalizers for transmultiplexers in orthogonal multiple carrier data transmission," in Proc. Eur. Signal Process. Conf., Trieste, Italy, Sep. 1996, vol. 2, pp. 1211-1214.

[10] G. Arslan, B. L. Evans, and S. Kiaei, "Equalization for discrete multitone receivers to maximize bit rate," IEEE Trans. Signal Process., vol. 49, no. 12 , pp. 3123-3135, Dec. 2001.

[11] R. K. Martin, K. Vanbleu, M. Ding, G. Ysebaert, M. Milosevic, B. L. Evans, M. Moonen, and C.R. Johnson, Jr., "Implementation complexity and communication performance tradeoffs in discrete multitone modulation equalizers," IEEE Trans. Signal Process., vol. 54, no. 5, pp. 3216-3230, Aug. 2006.

[12] K. Van Acker, G. Leus, M. Moonen, O. Van de Wiel, and T. Pollet, "Per tone equalization for DMT-based systems," IEEE Trans. Commun., vol. 49, no. 1, pp. 109-119, Jan. 2001

[13] Y. Gong and C. F. N. Cowan, "A novel variable tap-length algorithm for linear adaptive filters," in Proc. IEEE Int. Conf. Acoust., Speech, Signal Process., Montreal, QC, Canada, May 2004, vol. 2, pp. 825-828.

[14] F. Riera-Palou, J. M. Noras, and D. G. M. Cruickshank, "Variable length equalizers for broadband mobile systems," in Proc. 52nd IEEE Veh. Technol. Conf.-Fall, Boston, MA, Sep. 2000, vol. 5, pp. 2478-2485.

[15] B. Lu, L. D. Clark, G. Arslan, and B. L. Evans, "Fast time-domain equalization for discrete multitone modulation systems," in Proc. IEEE Digital Signal Process. Workshop, Hunt, TX, Oct. 2000, vol. 2, pp. 825-828.

[16] G. Arslan, B. Lu, L. D. Clark, and B. L. Evans, "Iterative refinement methods for time domain equalizer design," EURASIP J. Appl. Signal Process., vol. 2006, no. 7, pp. 47-58, Jul. 2006.

[17] A. M. Wyglinski, P. Kabal, and F. Labeau, "Adaptive filterbank multicarrier wireless systems for indoor environments," in Proc. 56th IEEE Veh. Technol. Conf.-Fall, Vancouver, BC, Canada, Sep. 2002, vol. 1, pp. 336-340.

[18] A. M. Wyglinski, P. Kabal, and F. Labeau, "Adaptive bit and power allocation for indoor wireless multicarrier systems," in Proc. 15th Int Conf. Wireless Commun., Calgary, AB, Canada, Jul. 2003, pp. 500-508.

[19] A. M. Wyglinski, F. Labeau, and P. Kabal, "Effects of imperfect subcarrier SNR information on adaptive bit loading algorithms for multicarrier systems," in Proc. IEEE Global Telecommun. Conf., Dallas, TX, Nov. 2004, pp. 3835-3839.

[20] B. Muquet, M. de Courville, and P. Duhamel, "Subspace-based blind and semi-blind channel estimation for OFDM systems," IEEE Trans. Signal Process., vol. 50, no. 7, pp. 1699-1712, Jul. 2002.

[21] G. Ysebaert, K. Vanbleu, G. Cuypers, M. Moonen, and T. Pollet, "Combined RLS-LMS initialization for per tone equalizers in DMT receivers," IEEE Trans. Signal Process., vol. 51, no. 7, pp. 1916-1927, Jul. 2003.

[22] A. Gersho and R. M. Gray, Vector Quantization and Signal Compression, ser. Communications and Information Theory. Norwell, MA: Kluwer, 1992.

[23] J. Medbo and P. Schramm, Channel models for HIPERLAN/2 in different indoor scenarios, Eur. Telecommun. Stand. Inst., ETSI EP BRAN Doc. 3ERI085B, 1998.

[24] E. A. Lee and D. G. Messerschmitt, Digital Communications, 2nd ed. Dordrecht, The Netherlands: Kluwer, 1994.

[25] M. Ding, B. L. Evans, and I. C. Wong, "Effect of channel estimation error on bit rate performance of time domain equalizers," in Proc. Asilomar Conf. Signals, Syst. Comput., Pacific Grove, CA, Nov. 2004, pp. 20562060. invited paper.

[26] T. Pollet and M. Peeters, "Synchronization with DMT modulation," IEEE Commun. Mag., vol. 37, no. 4, pp. 80-86, Apr. 1999.

[27] S. Haykin, Adaptive Filter Theory, 3rd ed. ser. Prentice-Hall Information and System Sciences. Englewood Cliffs, NJ: Prentice-Hall, 1996.

[28] T. K. Moon and W. C. Stirling, Mathematical Methods and Algorithms in Signal Processing. Englewood Cliffs, NJ: Prentice-Hall, 2000. 


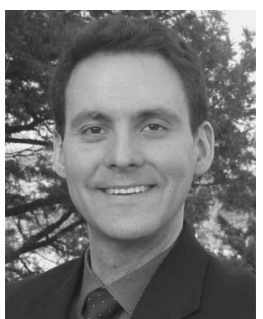

Alexander M. Wyglinski (S'99-M'05) received the B.Eng. degree from McGill University, Montreal, QC, Canada, in 1999, the M.S. degree from Queen's University, Kingston, ON, Canada, in 2000, and the $\mathrm{Ph} . \mathrm{D}$. degree from McGill University in 2005, all in electrical engineering.

He was an Assistant Research Professor with the Information and Telecommunication Technology Center, The University of Kansas, Lawrence. In August 2007, he joined the Department of Electrical and Computer Engineering, Worcester Polytechnic Institute, Worcester, MA, as an Assistant Professor. He is very actively involved in the wireless communications research community, particularly in the fields of cognitive radio systems and dynamic spectrum access networks. He was a Technical Program Committee Co-Chair for the Second International Conference on Cognitive Radio Oriented Wireless Networks and Communications (CrownCom 2007). His current research interests are wireless communications, wireless networks, cognitive radios, software-defined radios, transceiver optimization algorithms, dynamic spectrum access networks, spectrum sensing techniques, hybrid fiber-wireless networking, multihop and ad hoc networks, and signal processing techniques for digital communications.

Prof. Wyglinski currently serves on the Editorial Boards of both the IEEE Communications Magazine and the IEEE Communications Surveys and Tutorials. He served as a Guest Editor for the IEEE Communications Magazine Feature Topic on Cognitive Radio for Dynamic Spectrum Access in May 2007 and as a Track Chair for both the 64th and 66th IEEE Vehicular Technology Conferences. He is serving as a Technical Program Committee member on several IEEE and other international conferences in wireless communications and networks.

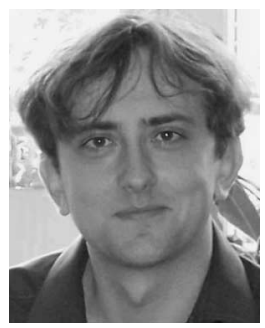

Martin Cudnoch received the B.Eng. (Honors) degree in electrical engineering, with specialization in telecommunications and control and automation, and the M.Eng. degree in electrical engineering, with specialization in telecommunications and signal processing, from McGill University, Montreal, QC, Canada, in 1999 and 2006, respectively.

During 1999-2003, he was an Applications Engineer with Teradyne Inc., Boston, MA. He is currently a DSP Engineer with SR Telecom Inc., Montreal, developing WiMAX technology. His research interests include adaptive OFDM transmission and algorithm optimization techniques.

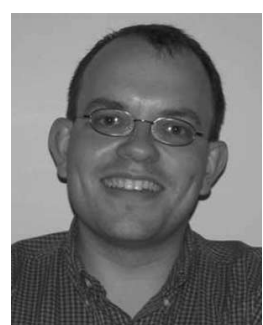

Fabrice Labeau (M'00-SM'07) received the Ph.D. degree from Université Catholique de Louvain, Louvain-la-Neuve, Belgium, in 2000.

Since 2000, he has been with the Department Electrical and Computer Engineering, McGill University, Montreal, QC, Canada, where he is currently an Associate Professor. He is also the Associate Director of the Centre for Advanced Systems and Technologies in Communications (SYTACom), McGill University. He has published more than 50 papers in refereed journals and conference proceedings. His research interests are signal processing for communications, transmission of compressed multimedia, and coding.

Dr. Labeau has served on the Technical Program Committees of many international conferences. He was the Local Arrangements Co-Chair for the 2004 IEEE International Conference on Acoustics, Speech, and Signal Processing and the Technical Program Committee Co-Chair for the Fall 2006 IEEE Vehicular Technology Conference.

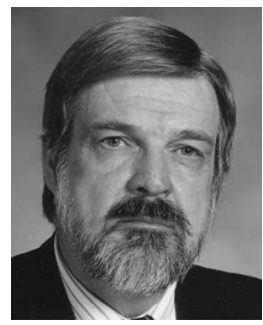

Peter Kabal (S'70-M'75) received the Ph.D. degree in electrical engineering from the University of Toronto, Toronto, ON, Canada, in 1975.

From 2000 to 2005, he was an NSERC/Nortel Industrial Research Chair. He is currently a Professor of electrical and computer engineering with the Department of Electrical and Computer Engineering, McGill University, Montreal, QC, Canada. His current research interests include digital signal processing applied to speech and audio processing, adaptive filtering, and data transmission. 